\title{
Hot-carrier-induced degradation of drain current hysteresis and transients in thin gate oxide floating body partially depleted SOI nMOSFETs
}

\author{
J.M. Rafi ${ }^{\mathrm{a}}$, E. Simoen ${ }^{\mathrm{b}}$, K. Hayama ${ }^{\mathrm{c}}$, A. Mercha ${ }^{\mathrm{b}}$, \\ F. Campabadal ${ }^{\mathrm{a}}, \mathrm{H}$. Ohyama ${ }^{\mathrm{c}}$, C. Claeys ${ }^{\mathrm{b}, \mathrm{d}}$ \\ ${ }^{a}$ Centro Nacional de Microelectrónica (IMB-CNM-CSIC), Campus UAB, 08193 Bellaterra (Barcelona), Spain \\ b IMEC, Kapeldreef 75, B-3001 Leuven, Belgium \\ ${ }^{\mathrm{c}}$ Kumamoto National College of Technology, 2569-2 Nishigoshi, Kumamoto 861-1102, Japan \\ ${ }^{\mathrm{d}}$ KU Leuven, Department of Electrical Engineering, Kasteelpark Arenberg 10, B-3001 Leuven, Belgium
}

\begin{abstract}
The impact of hot-carrier degradation on drain current $\left(I_{D}\right)$ hysteresis and switch-off $I_{D}$ transients of thin gate oxide floating body PD SOI nMOSFETs is analyzed. An extended characterization of these floating body effects (FBEs) is carried out for a wide range of transistor geometries and bias conditions. The results show a link between the hot-carrierinduced damage of the front channel and the reduction of the FBEs. This is further supported by unbiased thermal annealing experiments, which are found to give rise to a partial recovery of the hot-carrier induced damage and FBEs.
\end{abstract}

\section{Introduction}

In recent years, silicon on insulator (SOI) technologies have emerged as a serious competitor to bulk silicon technology. Owing to device integration requirements and intrinsic resistance constraints of the thin film layers, no film contacts are generally supplied. If body contacts are not present, so-called floating body effects (FBEs) arise, specially in partially depleted (PD) SOI MOSFETs, leading to some detrimental effects on device performance [1]. In particular, after switching on or off the front gate, there is no substrate current to quickly adjust the majority carrier density and equilibrium has to be reached through generation and recombination processes. Under these conditions, drain current $\left(\mathrm{I}_{\mathrm{D}}\right)$ transients [1] are observed, and these can be a useful tool for assessing the quality of the starting material, process-induced defects or contamination.
More recently, with the marked decrease in frontgate-oxide thickness, a new type of FBEs associated with gate-body direct tunneling has been identified $[2,3]$. For thin gate oxides (as thin as 2-2.5 nm), electron valence band (EVB) tunneling, occurring for a sufficiently large front-gate voltage $\left(\mathrm{V}_{\mathrm{FG}}\right)$ supplies majority carriers that charge the film and forward-bias the source-body junction, inducing a "kink" in the linear $\mathrm{I}_{\mathrm{D}}-\mathrm{V}_{\mathrm{FG}}$ characteristics and a second peak in the derived transconductance $\left(g_{\mathrm{mf}}\right)[2,3]$. Associated with this effect, a change in the $I_{D}$ transients shape is also observed $[2,3]$. The presence of these gate-induced FBEs (GIFBEs) [2] leading to history and memory effects, could be a limitation for partially depleted (PD) SOI CMOS circuit operation [4].

The problems associated with hot carriers (HC) have been recognized as one of the major constraints in silicon MOS scaling. Hot carriers are generated by the 
high lateral electric fields near the drain MOSFETs junction and may attain enough energy to overcome the $\mathrm{Si} /$ dielectric barrier, causing damage in this region, which leads to a gradual degradation of transistors current-voltage characteristics [5]. The damage is commonly believed to result in charge trapping in the gate dielectric and interface-state generation at the $\mathrm{Si} /$ dielectric interface.

In spite of the fact that FB SOI MOSFETs have been considered to be more resistant to $\mathrm{HC}$ degradation (HCD) than their bulk counterparts, what has been mainly attributed to a lower channel electric field [6], the presence of two different gates and their corresponding different interfaces makes HCD in SOI MOSFETs a more complex subject. In particular, opposite channel injection phenomena may occur [7], and, with the reduction in film thickness and fully depleted (FD) operation, coupling effects between the damage generated at the different gates [1]. More recently, with the introduction of very thin (below around $2.5 \mathrm{~nm}$ ) $\mathrm{SiO}_{2}$-based gate oxides, the high gate bias regimes have been shown to suffer from enhanced HCD [8,9], what has been attributed to the impact of the EVB direct tunneling injection phenomena, where the majority carriers introduced into the floating film have been reported to be responsible for this enhanced HCD [10].

In this study, we analyze the impact of hot-carrier degradation on drain current $\left(\mathrm{I}_{\mathrm{D}}\right)$ hysteresis and switchoff $\mathrm{I}_{\mathrm{D}}$ transients of thin gate oxide floating body PD SOI nMOSFETs. An extended characterization of these floating body effects is carried out for a wide range of transistor geometries and bias conditions. The results show the existence of a link between the hot-carrierinduced damage of the front channel and the reduction of the FBEs. This is further supported by unbiased thermal annealing experiments in the range of $150^{\circ} \mathrm{C}-250^{\circ} \mathrm{C}$, which are found to give rise to a partial recovery of the hot-carrier-induced damage and FBEs.

\section{Devices and experimental details}

The PD SOI nMOSFETs used in this study were fabricated in a $0.1 \mu \mathrm{m}$-CMOS process using a PELOX (polysilicon-encapsulated LOCOS) isolation and $2.5 \mathrm{~nm}$ nitrided gate oxide (NO). Processing was performed on $200 \mathrm{~mm}$ diameter UNIBOND wafers, with a final film thickness of $100 \mathrm{~nm}$. The buried oxide (BOX) thickness was $400 \mathrm{~nm}$. There is no film contact. Transistors with mask gate length $(\mathrm{L})$ ranging from $0.08 \mu \mathrm{m}$ to $10 \mu \mathrm{m}$ and width (W) from $0.2 \mu \mathrm{m}$ to $10 \mu \mathrm{m}$ were studied.
For the hot-carrier degradation experiments, the transistors were stressed at constant-voltage conditions and the degradation was mainly evaluated through the analysis of front and back channel input characteristics ( $I_{D}$ vs. $V_{F G}$ and $I_{D}$ vs. $V_{B G}$, respectively), which were regularly measured between the different stress phases. Owing to the floating body nature of the devices under study, special attention was paid to ensure that steadystate conditions were reached before performing the measurements after the different hot-carrier stress phases. A set of different front and back channel parameters, among them the front gate threshold voltage (defined as $\mathrm{V}_{\mathrm{T} f}=\mathrm{V}_{\mathrm{FG}} @ \mathrm{I}_{\mathrm{D}}=0.01 \mathrm{~W} / \mathrm{L} \mu \mathrm{A}$ ), were used as primary degradation monitors.

All current-voltage measurements and switch-off $\mathrm{I}_{\mathrm{D}}$ transients were measured using an HP-4155B Parameter Analyzer. During a conventional weak inversion singlegate switch-off experiment, the front gate is switched at $\mathrm{t}=0 \mathrm{~s}$ from an 'on' gate bias $\left(\mathrm{V}_{\mathrm{FGon}}\right)$ above $\mathrm{V}_{\mathrm{Tf}}$ to an 'off' gate bias ( $\left.\mathrm{V}_{\mathrm{FGoff}}\right)$ in the subthreshold regime [11]. Care was taken with the duration of the 'on' bias level, as well as for the conditions between two transient measurements, so that there were no device history (memory) effects. Unless, the contrary is indicated, the back gate (chuck contact to wafer substrate) was grounded and the drain bias was kept in the linear operation regime $\left(\mathrm{V}_{\mathrm{D}}=25 \mathrm{mV}\right)$. All experiments were carried out in a lightproof and electrically shielded setup.

\section{3. $I_{D}$ hysteresis characterization}

Figure 1 shows $\mathrm{I}_{\mathrm{D}}-\mathrm{V}_{\mathrm{FG}}$ and the derived $\mathrm{g}_{\mathrm{mf}}-\mathrm{V}_{\mathrm{FG}}$ characteristics corresponding to a floating body PD-SOI nMOSFET when $\mathrm{V}_{\mathrm{FG}}$ is swept from both, accumulation to inversion $\left(\mathrm{V}_{\mathrm{FG}}\right.$ sweep $\left.>0\right)$ and inversion to accumulation $\left(\mathrm{V}_{\mathrm{FG}}\right.$ sweep $\left.<0\right)$. It is known that $\mathrm{I}_{\mathrm{D}}$ hysteresis appears in these conditions, which is related to the floating body nature of the devices. The hysteresis can be defined as the $I_{D}$ difference between accumulation to inversion and inversion to accumulation $\mathrm{V}_{\mathrm{FG}}$ sweeps (Figure 1) [12].

The first peak in the $I_{D}$ hysteresis curve (positive hysteresis peak for $\mathrm{V}_{\mathrm{FG}}$ around $\mathrm{V}_{\mathrm{Tf}} \sim 0.5 \mathrm{~V}$ in Figure 1) is associated with the body potential difference between the switch-on and switch-off conditions present during the accumulation to inversion and inversion to accumulation $\mathrm{V}_{\mathrm{FG}}$ sweeps, respectively $[1,11]$. In the case of an nMOSFET, when $V_{F G}$ is swept from accumulation to inversion, holes have to be recombined to increase the space-charge depletion region for the new 


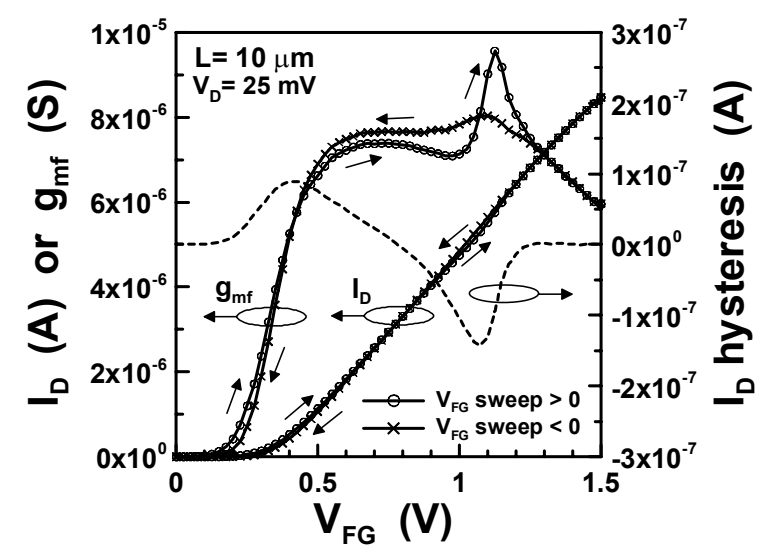

Figure 1. $\mathrm{I}_{\mathrm{D}}$ and $\mathrm{g}_{\mathrm{mf}} \mathrm{vs}$. $\mathrm{V}_{\mathrm{FG}}$ measured from accumulation to inversion $\left(\mathrm{V}_{\mathrm{FG}}\right.$ sweep $\left.>0\right)$ and inversion to accumulation $\left(\mathrm{V}_{\mathrm{FG}}\right.$ sweep $<0$ ) for an $\mathrm{L}=10 \mu \mathrm{m}$ nMOSFET. The corresponding $\mathrm{I}_{\mathrm{D}}$ hysteresis between the two sweep directions is also shown.

$\mathrm{V}_{\mathrm{FG}}$ condition. While this recombination process occurs, the $\mathrm{V}_{\mathrm{Tf}}$ of the device is slightly lower than the one that would correspond to such a $V_{F G}$ in steady state conditions. On the other hand, when $\mathrm{V}_{\mathrm{FG}}$ is swept from inversion to accumulation, holes have to be generated to replenish the space-charge region, during this generation process, $\mathrm{V}_{\mathrm{Tf}}$ is slightly higher than the one corresponding to steady state conditions $[1,11]$.

The second peak observed in the $\mathrm{I}_{\mathrm{D}}$ hysteresis curve (around $\mathrm{V}_{\mathrm{FG}}=1.1 \mathrm{~V}$ in Figure 1) is originated by the EVB tunneling phenomenon, which is characteristic of these thin gate oxide FB devices, and supplies majority carriers (holes) that charge the film and forward-bias the source-body junction, reducing $\mathrm{V}_{\mathrm{Tf}}$ and inducing a "kink" in the linear $\mathrm{I}_{\mathrm{D}}-\mathrm{V}_{\mathrm{FG}}$ characteristics and the $2^{\text {nd }}$ peak in the derived $g_{\mathrm{mf}}[2,3]$. In previous studies, the influence of different sweeping time steps on the GIFBEs [2,3], as well as the temperature dependence of the $I_{D}$ hysteresis peaks [12], have been pointed. Since time step issue is a critical point for FB devices, unless the opposite is indicated, a fixed $20 \mathrm{~ms}$ integration time was kept for all the $I_{D}$ double sweeps, which where performed at fixed room $\mathrm{T}$ conditions.

Originating from generation and recombination processes, the $I_{D}$ hysteresis, as well as the $I_{D}$ transients [11], can be a useful tool for assessing the quality of the starting material, process-induced defects, or contamination. An extended characterization of $I_{D}$ hysteresis was carried out for a wide range of transistor geometries ( $\mathrm{L}$ and $\mathrm{W}$ ) and bias conditions. The behavior of both, the conventional positive $1^{\text {st }}$ peak of $I_{D}$ hysteresis, as well as the EVB-induced negative $2^{\text {nd }}$ peak of $I_{D}$ hysteresis were studied. Figure 2 shows the dependence of both $I_{D}$ hysteresis peaks on transistor gate

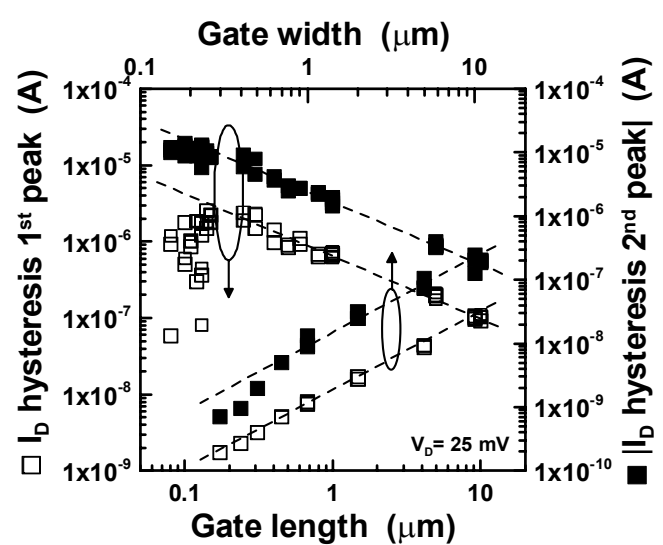

Figure 2. Dependence of the $1^{\text {st }}$ and $2^{\text {nd }}$ peaks of $I_{D}$ hysteresis (positive and negative, respectively) on transistor gate L and W.

$\mathrm{L}$ and $\mathrm{W}$. From the figure, it is appreciated an overall linear dependence of their magnitudes with $\mathrm{L}$ and $\mathrm{W}$, thus corroborating their expected scaling with channel current. In spite of the above general trends, the $1^{\text {st }}$ peak of $I_{D}$ hysteresis is found to be more sensitive to short channel effects, while the $2^{\text {nd }}$ peak of $\mathrm{I}_{\mathrm{D}}$ hysteresis seems to be more sensitive to narrow channel effects.

The decrease of the $1^{\text {st }} I_{D}$ hysteresis peak values for $\mathrm{L}$ below $0.20 \mu \mathrm{m}$ is thought to be originated by the influence of the corresponding nearby high negative $2^{\text {nd }}$ $\mathrm{I}_{\mathrm{D}}$ hysteresis peak. The fact that these devices received a halo (or pocket) source/drain extensions implantation (to suppress short channel effects) makes their $\mathrm{V}_{\text {Tf }}$ to slightly increase for the shorter L (reverse short channel effect). In this way, $\mathrm{V}_{\mathrm{Tf}}$ of an $\mathrm{L}=0.1 \mu \mathrm{m} \mathrm{nMOSFET}$ is about 0.2 $\mathrm{V}$ higher than the corresponding value for an $\mathrm{L}=10 \mu \mathrm{m}$ device [13]. The higher position of $\mathrm{V}_{\mathrm{Tf}}$, as well as the increasing magnitude of the EVB-related negative $2^{\text {nd }}$ peak of $I_{D}$ hysteresis, is thought to lead to some impact on the values for the $1^{\text {st }}$ peak of $I_{D}$ hysteresis in the shorter devices $(\mathrm{L}<\sim 0.2 \mu \mathrm{m})$. In fact, as it will be shown later, this phenomenon can be also observed in the case of HC-stressed short L devices, in which a HCinduced positive $\Delta \mathrm{V}_{\mathrm{Tf}}$ can also lead to a higher degree of overlap between the two $I_{D}$ hysteresis peaks. From the above, it is inferred that the $1^{\text {st }}$ peak of $\mathrm{I}_{\mathrm{D}}$ hysteresis may not be a very suitable parameter for assessment of FBEs degradation in short-L nMOSFETs.

It should be also commented that the sensitivity to narrow channel effects of the EVB-related $2{ }^{\text {nd }}$ peak of $I_{D}$ hysteresis can be associated with the higher generation/recombination rates expected near the isolation, which leads to reduced FBEs [1]. Regarding to 
the other remaining $\mathrm{L}$ and $\mathrm{W}$ dependences, only a saturation of the $2^{\text {nd }} I_{D}$ hysteresis peak is observed in the short $\mathrm{L}$ region, which is also observed for the $\mathrm{g}_{\mathrm{mf}}$ peaks [14]. On the other hand, the narrow channel effects seem not to affect the conventional $1^{\text {st }}$ peak of $\mathrm{I}_{\mathrm{D}}$ hysteresis, as they also do not do for $\mathrm{V}_{\mathrm{Tf}}$.

\section{Hot-carrier degradation}

A set of transistors were subjected to constantvoltage $\mathrm{HC}$ stress under different $\mathrm{V}_{\mathrm{D}}$ and $\mathrm{V}_{\mathrm{FG}}$ conditions. A particular HCD mode, characterized by a positive front gate threshold voltage shift with a turn-around behavior for long term stress was observed [9]. A significant negative charge trapping in the back gate was also observed after long HC stress. However, in this study, we concentrate on the investigation of the degradation of two main FBEs, which are the $I_{D}$ hysteresis and the switch-off $\mathrm{I}_{\mathrm{D}}$ transients. For this purpose, the results shown in this chapter will mainly refer to the first phases of the HCD (that is, for low stress conditions that correspond to either low stress voltages or times and/or longer channel devices), in which the damage is mainly limited to the front channel [9]. For harder stress conditions, the damage also affects the back channel and a more dedicated analysis is needed, in particular, under such higher damage conditions the switch-off $\mathrm{I}_{\mathrm{D}}$ transients rapidly vanish [9].

\subsection{Hot-carrier degradation of $I_{D}$ hysteresis}

Figure 3 shows a typical example of the $I_{D}$ hysteresis measured for an nMOSFET after different HCD stress times. A clear decrease of the magnitude of the positive and negative $I_{D}$ hysteresis peaks is observed. Moreover, a significant $\mathrm{V}_{\mathrm{FG}}$-shift is observed for the conventional positive $\mathrm{I}_{\mathrm{D}}$ hysteresis peak, which can be associated with the $\mathrm{HC}$-induced $\Delta \mathrm{V}_{\mathrm{Tf}}$ experimented by the devices. However, no such a behavior is observed for the case of the EVB-related $2^{\text {nd }}$ peak of the $I_{D}$ hysteresis, thus leading to a higher degree of overlap between the two $\mathrm{I}_{\mathrm{D}}$ hysteresis peaks after hot-carrier degradation.

In the case of FB SOI MOSFETs under low $\mathrm{V}_{\mathrm{D}}$ conditions, it is considered that the dominating generation and recombination mechanisms can occur in four main regions: the channel space-charge and film region, the source and drain junctions, and the front and back channel interfaces [15].

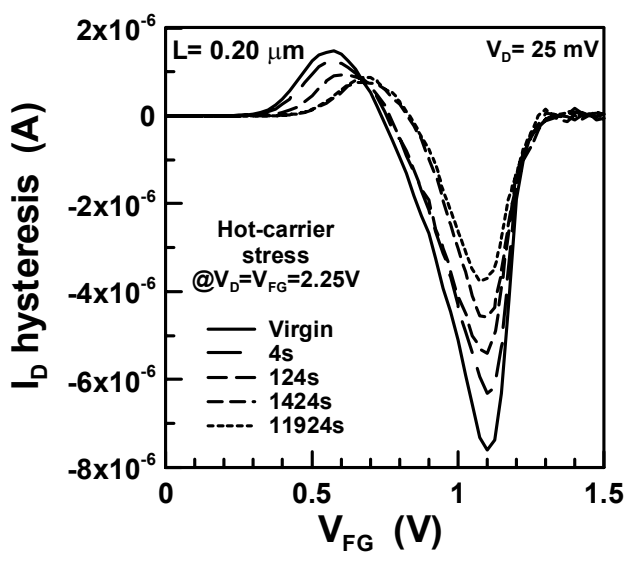

Figure 3. ID hysteresis measured for an nMOSFET before hotcarrier degradation and after different stress times.

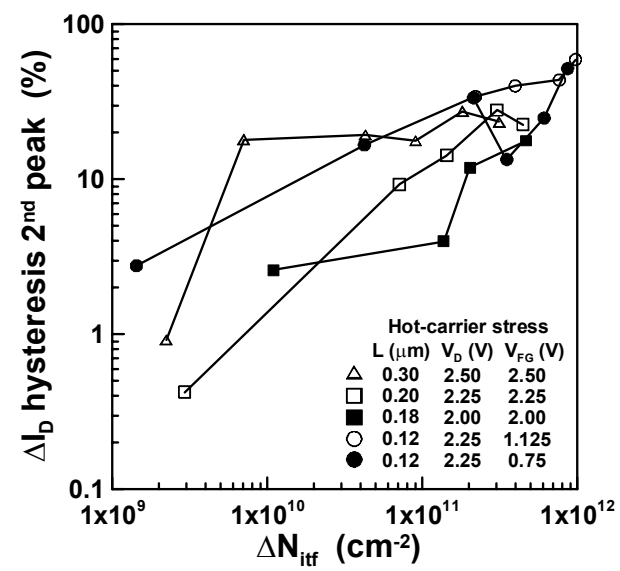

Figure 4. Degradation of $2^{\text {nd }}$ peak of $I_{D}$ hysteresis vs. front channel density of interface states generated during $\mathrm{HC}$ stress of different nMOSFETs (extracted according to [16]). $\left(\Delta \mathrm{I}_{\mathrm{D}}\right.$ hysteresis is given in percentage ( $\mathrm{I}_{\text {Dhysteresis }}{ }^{-}$ $\left.\mathrm{I}_{\text {Dhysteresis0 }}\right) / \mathrm{I}_{\text {Dhysteresis0 }}$, where $\mathrm{I}_{\text {Dhysteresis } 0}$ is before stress value).

In order to evaluate a possible correlation between the $\mathrm{HC}$-induced damage and the observed $\mathrm{I}_{\mathrm{D}}$ hysteresis behavior, figure 4 shows the dependence of the $2^{\text {nd }}$ peak of $I_{D}$ hysteresis on the increase of the front channel density of interface states $\left(\Delta \mathrm{N}_{\mathrm{itf}}\right)$ generated during the HC stress of different nMOSFETs. Although a slight scattering of the $I_{D}$ hysteresis peak values can be sometimes observed (what can be attributed to experimental conditions and the use of such a short $\mathrm{L}$ devices, for which the $2^{\text {nd }}$ peak of $I_{D}$ hysteresis can be affected by geometrical factors (Figure 2)), an overall correlation between the $\mathrm{HC}$-induced damage and the reduction of the $I_{D}$ hysteresis can be appreciated. 


\subsection{Hot-carrier degradation of switch-off $I_{D}$ transients}

In order to investigate the impact of HCD on generation and recombination $\mathrm{I}_{D}$ transients, front gate switch-off $I_{D}$ transients were measured for the nMOSFETs before and after HCD, using $\mathrm{V}_{\mathrm{FGon}}$ voltages above and below the $\mathrm{V}_{\mathrm{FG}}$ threshold voltage for body to gate EVB tunneling [11]. The results after HCD showed faster generation and recombination processes in the film (Figure 5), with a correlation between the HCD of the front channel and the reduction of this FBE. This suggests an increased contribution of surface states on the generation and recombination processes [17].

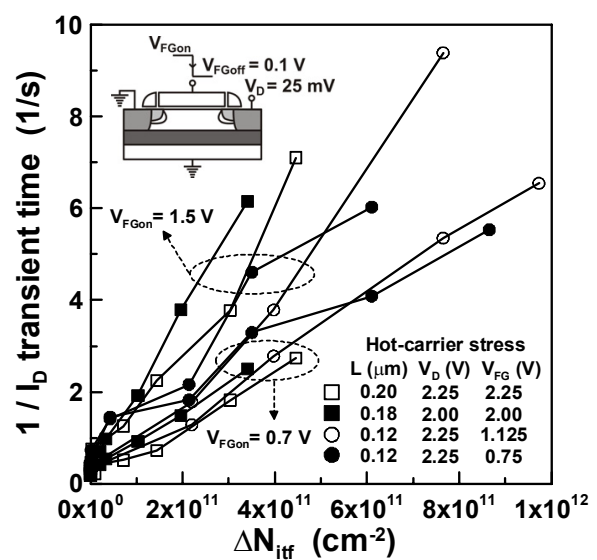

Figure 5. Dependence of the inverse of switch-off $I_{D}$ transient times (defined as the time to reach either, $90 \%$ (undershoot: $\mathrm{V}_{\mathrm{FGon}}=0.7 \mathrm{~V}$ ) or $110 \%$ (overshoot: $\mathrm{V}_{\mathrm{FGon}}=1.5 \mathrm{~V}$ ) of $\mathrm{I}_{\mathrm{D}}(\mathrm{t}=\infty)$ ) on the front channel density of interface states generated during the HCD of different nMOSFETs (extracted according to [16]).

\section{Thermal annealing effects}

In order to gain better insight into the $\mathrm{HC}$-induced damage affecting the FBEs and evaluate its thermal stability, some annealing experiments were carried out by applying different constant temperature annealing phases in the range of $150-250{ }^{\circ} \mathrm{C}$ to previously $\mathrm{HC}$ stressed nMOSFETs. During the thermal anneals, the device terminals were left floating and care was taken in order to get enough cooling of the samples down to room $\mathrm{T}$ before the measurements were performed.

Figure 6 shows a typical example of the evolution of the $1^{\text {st }}$ and $2^{\text {nd }}$ peaks of $\mathrm{I}_{\mathrm{D}}$ hysteresis for two nMOSFETs subjected to different hot-carrier stress times and subsequent consecutive thermal annealing phases. In figure 7 there are given the corresponding $\mathrm{V}_{\mathrm{FG}}$ values at which the $\mathrm{I}_{\mathrm{D}}$ hysteresis peaks were registered.

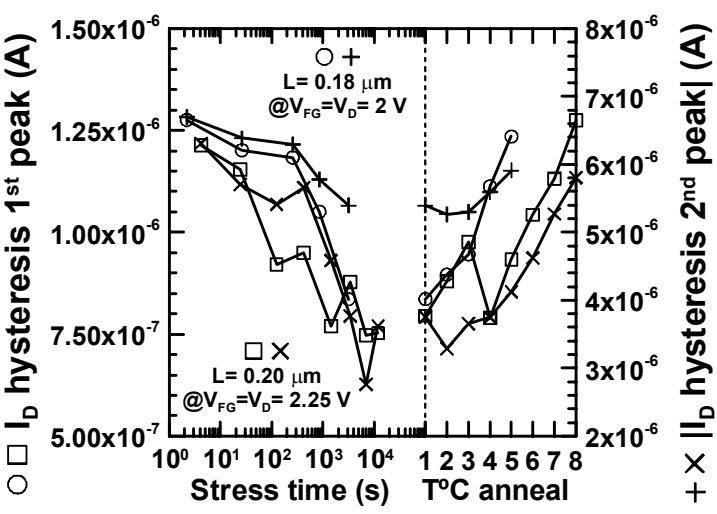

Figure 6. Evolution of $I_{D}$ hysteresis peaks for two nMOSFETs subjected to HCD and subsequent consecutive thermal annealing phases $\left(\mathrm{T}^{\circ} \mathrm{C}\right.$ anneal for $\mathrm{L}=0.18 \mu \mathrm{m}$ device: 1 : Just after the HC stress, 2:+1000s @ $145^{\circ} \mathrm{C}, 3:+1000 \mathrm{~s} @ 175^{\circ} \mathrm{C}, 4$ : +1000s@195 $\left.{ }^{\circ} \mathrm{C}, 5:+1000 \mathrm{~s} @ 225^{\circ} \mathrm{C}, 6:+2000 \mathrm{~s} @ 250^{\circ} \mathrm{C}\right)$. $\left(\mathrm{T}^{\circ} \mathrm{C}\right.$ anneal for $\mathrm{L}=0.20 \mu \mathrm{m}$ device: 1 : re-measured after $\mathrm{HCD}$ and 25 days at room T, 2: $+600 \mathrm{~s} @ 145^{\circ} \mathrm{C}, 3:+600 \mathrm{~s} @ 165^{\circ} \mathrm{C}$, 4:+600s@170 ${ }^{\circ} \mathrm{C}, 5:+900 \mathrm{~s} @ 190^{\circ} \mathrm{C}, 6:+900 \mathrm{~s} @ 195^{\circ} \mathrm{C}, 7$ :

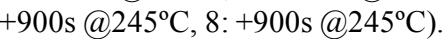

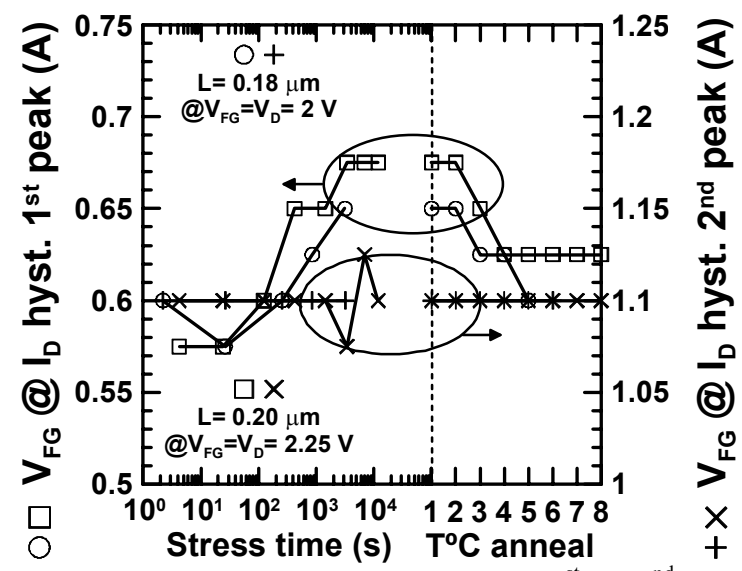

Figure 7. Evolution of the $V_{F G}$ at which the $1^{\text {st }}$ and $2^{\text {nd }}$ peaks of $I_{D}$ hysteresis are registered for figure 6 nMOSFETs.

From figures 6 and 7, it is clearly observed that unbiased thermal annealing experiments lead to a partial recovery of both, the $I_{D}$ hysteresis peaks, as well as the $\mathrm{V}_{\mathrm{FG}}$ values at which the $1^{\text {st }}$ peak is registered. This is further supported by the results from switch-off $I_{D}$ transients (not shown here), in which both, the steadystate $I_{D}$ levels, as well as the transients times, were also partially recovered after unbiased thermal annealing experiments. Finally, figure 8 shows the evolution of the extracted front channel oxide trapped charges and interface states densities for figures 6 and 7 nMOSFETs. The thermal annealing experiments were found to give rise to a clear partial recovery of the hot-carrier-induced 
damage, thus supporting the existence of a link between the physical hot-carrier-induced damage of the front channel and the reduction of the FBEs.



Figure 8. Evolution of front channel oxide trapped charges and interface states densities $\left(\mathrm{N}_{\text {otf }}\right.$ and $\mathrm{N}_{\text {itf }}$, extracted according to [16]) for figure $6 \mathrm{nMOSFETs.}$

\section{Conclusions}

The impact of hot-carrier degradation on ID hysteresis and switch-off ID transients of thin gate oxide floating body PD SOI nMOSFETs is analyzed. An extended characterization of these floating body effects (FBEs) is carried out for a wide range of transistor geometries and bias conditions. The results show a link between the hot-carrier-induced damage of the front channel and the reduction of the FBEs. This is further supported by unbiased thermal annealing experiments in the range of $150^{\circ} \mathrm{C}-250^{\circ} \mathrm{C}$, which are found to give rise to a partial recovery of the hot-carrier-induced damage and FBEs.

\section{Acknowledgements}

J.M. Rafi acknowledges the EC for his post-doc Marie Curie European Reintegration Grant and the Spanish Ministry of Science and Technology for the Ramón y Cajal Program.

\section{References}

[1] Cristoloveanu S, Li SS. Electrical Characterization of Silicon-On-Insulator Materials and Devices. Boston: Kluwer Academic Publishers; 1995.

[2] Pretet J, Matsumoto T, Poiroux T, Cristoloveanu S, Gwoziecki R, Raynaud C, et al. New mechanism of body charging in partially depleted SOI-MOSFETs with ultrathin gate oxides. Proc. of ESSDERC, 2002, p. 515-8.

[3] Mercha A, Rafí JM, Simoen E, Augendre E, Claeys C. "Linear Kink Effect" induced by valence band electron tunneling in ultra-thin gate oxide bulk and SOI MOSFETs. IEEE Trans Electron Dev 2003;50:1675-82.

[4] Yang J-W, Fossum JG, Workman GO, Huang C-L. A physical model for gate-to-body tunneling current and its effects on floating-body PD/SOI CMOS devices and circuits. Solid-State Electron 2004;48:259-70.

[5] Hu C, Tam SC, Hsu F-C, Ko P-K, Chan T-Y, Terrill KW. Hot-electron-induced MOSFET degradation - model, monitor and improvement. IEEE Trans Electron Dev 1985;32:375-85.

[6] Colinge J-P. Hot-electron effects in silicon-on-insulator nchannel MOSFET's. IEEE Trans Electron Dev 1987;ED34:2173-7.

[7] Ioannou DE, Duan FL, Sinha SP, Zaleski A. Oppositechannel-based injection of hot-carriers in SOI MOSFET's: physics and applications. IEEE Trans Electron Dev 1998;45:1147-54.

[8] Dieudonné F, Haendler S, Jomaah J, Balestra F. Low frequency noise and hot-carrier reliability in advanced SOI MOSFETs. Solid-State Electron 2004;48:985-97.

[9] Rafí JM, Simoen E, Mercha A, Campabadal F, Claeys C. Impact of Hot-Carrier Stress on Gate-Induced Floating Body Effects and Drain Current Transients of Thin-GateOxide Partially Depleted SOI nMOSFETs. Solid-State Electron 2005;49:1536-46.

[10] Tsai CW, Chen MC, Ku SH, Wang T. Auger recombination-enhanced hot carrier degradation in nMOSFETs with a forward substrate bias. IEEE Trans Electron Dev 2003;50:1022-6.

[11] Rafí JM, Mercha A, Simoen E, Claeys C. Impact of gate tunneling floating-body charging on drain current transients of $0.10 \mu \mathrm{m}$-CMOS partially depleted SOI MOSFETs. Solid-State Electron 2004;48:1211-21.

[12] Hayama K, Takakura K, Okada S, Kudou T, Ohyama H, Rafí JM, et al. Investigation of back gate interface states by drain current hysteresis in PD-SOI n-MOSFETs. Physica B 2006;376-377:416-9.

[13] Simoen E, Rafí JM, Mercha A, Claeys C. Total ionizing dose damage in deep submicron partially depleted SOI MOSFETs induced by proton irradiation. Solid-State Electron 2004;48:1045-54.

[14] Rafi JM, Mercha A, Simoen E, Claeys C, Mohammadzadeh A. Radiation-Induced Back Channel Leakage in $60 \mathrm{MeV}$-Proton-Irradiated $0.10 \mu \mathrm{m}$-CMOS Partially Depleted SOI MOSFETs. Proc. of RADECS, 2003, 425-32.

[15] Shin H, Racanelli M, Huang WM, Foerstner J, Hwang T, Schroder DK. Measurement of carrier generation lifetime in SOI devices. Solid-State Electron 1999;43:349-53.

[16] McWhorter PJ, Winokur PS. Simple technique for separating the effects of interface traps and trapped-oxide charge in metal-oxide-semiconductor transistors. Appl Phys Lett 1986;48:133-5.

[17] Kimpton D, Kerr J. Generation lifetime, interface state density, active defect density and oxide resistivity measurements for SOI-MOSFETs and their radiation dependence. IEEE Trans Nucl Sci 1992;39:2126-31. 